\section{Wasserkraft - innovative Ansätze zur technischen, ökonomischen und ökologischen Optimierung}

In Österreich deckt die Wasserkraft derzeit etwa 58,5\% des Bedarfs an elektrischer Energie (E-Control, 2011) ab und ist ein damit bedeutender Sektor der österreichischen Energiewirtschaft. Viele der existierenden über 5200 Wasserkraftwerke entsprechen u. a. aufgrund geänderter gesetzlicher Vorgaben nicht mehr dem Stand der Technik und bedürfen einer Optimierung. Weiters besteht gemäß Energiestrategie Österreich ein Ausbauziel von 3,5 Terrawattstunden (TWh) bis 2015. 0,7 TWh können davon durch Effizienzsteigerungen und Revitalisierungen bestehender Standorte erreicht werden. Die simultane Erfüllung der Anforderungen aus der Wasserrahmenrichtlinie, der Erneuerbaren-Energien-Richtlinie sowie infolge technischer Neuerungen und variabler energiewirtschaftlicher Rahmenbedingungen am Strommarkt erfordern innovative Ansätze zur technischen, ökonomischen und ökologischen Optimierung der Wasserkraft bei Revitalisierung und Neubau.

Im vorliegenden Heft finden sich fünf Artikel zum Thema Wasserkraft, die den Bogen von technischen, ökonomischen bis zu ökologischen Optimierungen und integrativen Ansätzen spannen.

Der erste Artikel „Optimierung von Wasserkraft und Ökologie bei Erneuerung oder Revitalisierung bestehender Anlagen - ,win-win“-Ansatz“ (Habersack et al.) stellt einen Lösungsansatz zur simultanen Verbesserung von Wasserkraft und Ökologie vor. Der im Projekt Nachhaltige Entwicklung der Kamptal-Flusslandschaft entwickelte „win-win“-Ansatz kann generell bei der Optimierung, Effizienzsteigerung und Revitalisierung von bestehenden Wasserkraftanlagen zum Einsatz kommen, um einerseits mehr Strom zu erzeugen und andererseits z.B. mehr Restwasser zur Verbesserung des ökologischen Zustands abzugeben.

Im Artikel „Grundlagenuntersuchungen und Methodikentwicklung zur Bewertung des WasserkraftSchwalls bei unterschiedlichen Flusstypen“ (Hauer et al.) bilden die flusstypspezifischen Eigenschaften und deren Wechselwirkung mit Schwall- und Sunkprozessen den Kern der Arbeit, welche die wesentlichsten Ergebnisse des Forschungsprojekts SCHWALL_2012 zusammenfasst. Die Ergebnisse zeigen, dass eine flusstypspezifische, auch streckenabhängige Bewertung des Sunk-Schwalleinflusses für ein zukünftiges Management als notwendig betrachtet wird, da entsprechend der jeweiligen Gewässermorphologie unterschiedliche Habitatveränderungen in Abhängigkeit von der saisonalen Wasserführung auftreten können.

Der Artikel „Die Wasserkraftschnecke - Praxis, Prüfstand und Potenzial“ (Lashofer et al.) beschäftigt sich mit Wasserkraftschnecken und fasst auf Basis einer Betreiberbefragung, umfangreichen Feldmessungen und Laborversuchen den Entwicklungsstand zusammen. In umfangreichen Labormessungen wurden Wirkungsgrade für sieben Schneckentypen bei unterschiedlichsten Drehzahl/Durchfluss-Kombinationen und Achsneigungen gemessen und verglichen. Damit zeigen sich sowohl ausgezeichnete Wirkungsgrade als auch die unterschiedlichen Auswirkungen von veränderten Gestaltparametern. Gleichzeitig konnte weiteres Verbesserungspotenzial zum Stand der Technik dargestellt werden.

Gabl et al. zeigen im Artikel „Numerische Berechnung von Hochdruckanlagen: Global betrachtet lokal verbessert“, dass durch die Verwendung von zusätzlichen 3D-numerischen Untersuchungen die hydraulischen Berechnungen des Triebwasserweges von Hochdruckwasserkraftanlagen verbessert werden. Anhand von drei Beispielen werden exemplarisch die Möglichkeiten aufgezeigt, welche sich durch eine solche vertiefte Betrachtung ergeben. Dabei handelt es sich um eine Oberkammer, eine asymmetrische Düse und eine Anbindung eines sich im Bau befindenden neuen Teils einer Wasserkraftanlage mit einem bestehenden Druckstollen.

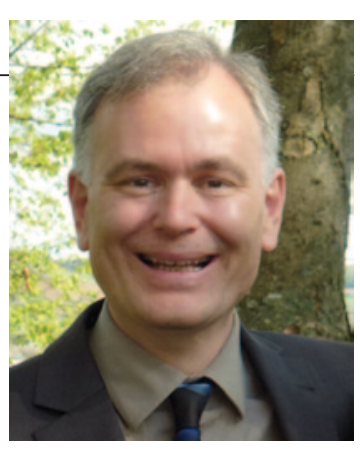

Univ.-Prof. DI Dr. Helmut Habersack 
Im Artikel „Die Bedeutung von Feststoffhaushalt und Sedimentdurchgängigkeit für eine nachhaltige Nutzung der Wasserkraft" greifen Habersack et al. ein zunehmend wichtigeres Thema im Kontext der Wasserkraftnutzung auf. Die Frage des Sedimentkontinuums sollte bei der Neuerrichtung bzw. beim Umbau bestehender Wasserkraftwerke berücksichtigt werden. Ein vorgeschlagener Lösungsansatz basiert auf einer integralen Betrachtung, wo eine Optimierung des Stauraummanagements aus technischer, ökonomischer und ökologischer Sicht unter Einbeziehung der verschiedenen betroffenen StakeholderInnen erfolgt und der die vielfältigen natürlichen Prozesse (Erosion, Transfer, Sedimentation, Remobilisierung) involviert.

\section{Univ.-Prof. DI Dr. H. Habersack}

Leiter Christian Doppler Labor für Innovative Methoden in

Fließgewässermonitoring, Modellierung und Flussbau

Leiter Institut für Wasserwirtschaft, Hydrologie und konstruktiven Wasserbau

Department Wasser - Atmosphäre - Umwelt

Universität für Bodenkultur Wien, Muthgasse 107, 1190 Wien, Austria

helmut.habersack@boku.ac.at 